\title{
Intervenciones para dejar de fumar en México: análisis de disponibilidad a pagar por un método efectivo de cesación
}

\author{
Edson E Serván-Mori, MsC,(1) lleana B Heredia-Pi, PhD,(2) \\ Luz Myriam Reynales-Shigematsu, PhD, (3) Sergio Bautista-Arredondo, MsC.(I)
}

\begin{abstract}
Serván-Mori EE, Heredia-Pi IB, Reynales-Shigematsu LM, Bautista-Arredondo S. Intervenciones para dejar de fumar en México: análisis de disponibilidad a pagar por un método efectivo de cesación. Salud Publica Mex 20 12;54:213-224.
\end{abstract}

\begin{abstract}
Resumen
Objetivo. Identificar factores socioeconómicos, demográficos, historia de tabaquismo y contextuales asociados con el deseo de dejar de fumar, estimar la disponibilidad a pagar (DAP) por tratamientos de cesación tabáquica (TCT) efectivos, e identificar sus factores asociados. Material y métodos. Mediante la Encuesta Global de Tabaquismo en Adultos, México 2009, caracterizamos a I 626 fumadores. Modelos logit y de regresión lineal múltiple permitieron identificar factores asociados con el deseo de dejar de fumar y la DAP. Resultados. $82.2 \%$ de los fumadores que no deseaban dejar de fumar fueron hombres. Entre quienes deseaban dejar de fumar, $49.8 \%$ fumaba diariamente $y$ reportó más de 16 años de fumar, $57 \%$ manifestó intentos previos de cesación y $10 \%$ conocer centros de ayuda. La DAP promedio fue $2708 \mathrm{MXN}$, destacando diferencias por nivel socioeconómico y educativo. Conclusiones. Se contribuye al diseño de estrategias de cesación diferenciadas, propiciando mejoras en la respuesta del sistema de salud al combate del tabaquismo en México.
\end{abstract}

Palabras clave: tabaquismo; cesación tabáquica; análisis costobeneficio; valoración contingente; México
Serván-Mori EE, Heredia-Pi IB,

Reynales-Shigematsu LM, Bautista-Arredondo S.

Smoking cessation interventions in Mexico:

Analysis of the willingness to pay

for an effective method to quit.

Salud Publica Mex 20I2;54:2 I3-224.

\begin{abstract}
Objective. To identify environmental, demographic and socioeconomic factors associated with the desire to quit, estimate the willingness to pay (WTP) for smoking cessation treatments (SCT) and to identify associated factors with this valuation. Materials and methods. Using the Global Adult Tobacco Survey, Mexico 2009, we characterized I 626 smokers. Logistic and multiple lineal regression models allowed to identify associated factors with the desire to quit and the WTP for SCT. Results. $82.2 \%$ of the current smokers who did not want to quit were men. Between those who wanted to quit, $49.8 \%$ had been consuming tobacco every day, for more than 16 years, $57 \%$ had made cessation attempts in the past, and around $10 \%$ knew about the existence of centers to help quit smoking. Average WTP was 2708 Mexican pesos (MXP), with differences by educational and socioeconomic levels. Conclusions. This evidence supports policymakers in the design of smoking cessation interventions improving national health system interventions for quit smoking.
\end{abstract}

Key words: smoking; smoking cessation; cost benefit analysis; contingent valuation; Mexico

(I) Dirección de Economía de la Salud, Centro de Investigación en Evaluación y Encuestas, Instituto Nacional de Salud Pública. Cuernavaca, México.

(2) Dirección de Gerencia y Liderazgo en Salud, Centro de Investigación en Sistemas de Salud, Instituto Nacional de Salud Pública. Cuernavaca, México.

(3) Grupo de Investigación sobre el Tabaco, Centro de Investigación en Salud Poblacional, Instituto Nacional de Salud Pública. Cuernavaca, México. 
$E^{1}$ apoyo a la cesación tabáquica es una de las cinco áreas de intervención principales en el combate de la epidemia de tabaquismo, establecidas en el Convenio Marco para el Control del Tabaco $(\mathrm{CMCT})^{1}$ y en las estrategias MPOWER de la Organización Mundial de la Salud (OMS). ${ }^{2}$ En México, actualmente, existen varios tratamientos de cesación tabáquica (TCT) eficaces, tanto de índole psicológica como farmacológica, como la terapia de reemplazo de nicotina (parches, goma de mascar y los inhaladores de nicotina), y los compuestos no nicotínicos como el bupropion, la nortriptilina y la vareniclina. ${ }^{3-6}$

En el ámbito internacional, cerca de $70 \%$ de los fumadores quiere dejar de fumar, 30\% ha hecho algún intento previo de cesación y de éstos $8 \%$ ha utilizado algún medicamento para dejar de fumar. ${ }^{7}$ En México, la Encuesta Nacional de Adicciones $2008^{8}$ informó que $51.3 \%$ de los fumadores ha intentado dejar de fumar alguna vez, sin embargo, sólo $2.6 \%$ de ellos se ha sometido a algún TCT, y aunque $72 \%$ de los fumadores ha escuchado hablar sobre algún medicamento que ayuda a dejar de fumar, $37.1 \%$ opina que son muy caros; $29.5 \%$ que pueden dañar la salud, y $16.9 \%$ que son difíciles de conseguir. ${ }^{8}$ Esta información muestra el enorme espacio de oportunidad para la implementación de intervenciones que apoyen a los fumadores en su intento por dejar de fumar.

Adicionalmente, datos de México sugieren que, si bien más de la mitad de los fumadores desea abandonar el consumo de productos de tabaco, un porcentaje importante lo intenta sin apoyo psicológico, médico, ni farmacológico. ${ }^{9}$ Los fumadores mexicanos muestran niveles de utilización de los métodos de cesación aún muy bajos. ${ }^{9}$ Ello se vuelve más relevante si consideramos informes de otros contextos que señalan que menos de $3 \%$ de los fumadores que hacen un intento por dejar de fumar lo logran únicamente mediante la fuerza de voluntad. ${ }^{10-13}$ Lo anterior se puede explicar, al menos en parte, por la existencia de importantes barreras al acceso y utilización de los programas de cesación, por el nivel de información sobre los métodos para dejar de fumar, por la deficiencia en la oferta oportuna de estos servicios y por la falta de capacidad de pago por parte del fumador.

Un fumador preparado para dejar de fumar, al ser informado de los potenciales beneficios de la cesación -tanto para él como para terceros- puede revelar sus preferencias por un método de cesación que le ofrezca tales beneficios, asignando un valor monetario a dicho método. Esta información además puede ser utilizada para aproximar la demanda por dichos servicios y ser de utilidad para los tomadores de decisiones para evaluar la oferta de los TCT ofrecidos por el sistema nacional de salud y para valorar potenciales estrategias de financiamiento de las mismas.

La metodología de la disponibilidad a pagar (DAP) es una herramienta diseñada para este tipo de análisis. ${ }^{14-}$ ${ }^{18}$ Esta metodología requiere que cada sujeto de estudio responda a preguntas hipotéticas cuyo objetivo es obtener el valor social, en términos monetarios, de una intervención específica.

En el área de la cesación tabáquica, la evidencia existente al respecto es escasa, sin embargo hay ejercicios previos relevantes. Por ejemplo, Bush y cols, ${ }^{19}$ exploraron la factibilidad de la utilización de la técnica para valorar un hipotético y novedoso TCT, demostrando que existe un mercado potencialmente sustancial para los TCT, especialmente frente a incrementos en su efectividad en comparación con los ya existentes. Los autores incluyeron en sus análisis variables relacionadas con las características socioeconómicas y demográficas de los entrevistados (edad, sexo, raza, nivel educativo e ingreso familiar), con su historia de fumador (número de intentos previos para dejar de fumar, número de cigarrillos consumidos al día) y variables relacionadas con el peso corporal y antecedentes de alcoholismo (índice de masa corporal, antecedentes de intentos por bajar de peso e intensidad de la dependencia al alcohol). En este estudio se demostró una mayor DAP entre fumadores del sexo femenino y en los encuestados con un nivel socioeconómico más alto. Ninguna de las variables relacionadas con la historia de fumador fueron encontradas asociadas de manera estadísticamente significativa con la DAP por un método de cesación efectivo. ${ }^{19}$

En otro estudio, Weimer y cols, ${ }^{20}$ utilizaron la metodología DAP para estimar la valoración por los fumadores de un TCT. Para ello midieron la pérdida en el excedente del consumidor, derivada de la reducción en el consumo de productos de tabaco por la implementación de políticas de combate al tabaquismo. Propusieron una novedosa forma de estimar el costo social de las intervenciones de cesación, argumentando que la forma correcta de medir los cambios en el bienestar individual, a partir de intervenciones para dejar de fumar, sólo debería considerar bienes no adictivos y no la demanda de los bienes adictivos. Estos hallazgos cambiaron considerablemente la valoración de las ventajas netas de implementar programas y políticas que intentan reducir el consumo de bienes adictivos. ${ }^{20}$ Los modelos estimados por los autores sugieren asociaciones estadísticamente significativas entre la DAP y las variables de ingreso, intensidad del consumo, gasto en los productos de tabaco, intentos previos de cesación, percepción sobre las consecuencias para la salud por el consumo de cigarrillos y el grado de dependencia física y psicológica a la nicotina. ${ }^{20}$ 
En el caso de México, se han reportado algunos estudios que aplican la metodología de la DAP en el campo de la salud pública, pero no relacionados con el área de la cesación tabáquica. ${ }^{21-23}$ Sin embargo un estudio reciente aplicó la metodología de la DAP al área específica del control de la epidemia de tabaquismo. ${ }^{*}$ Se trata de un estudio realizado a partir de la Encuesta Global de Tabaquismo en Adultos, México 2009. En el mismo, los autores estimaron la DAP de los fumadores para tres diferentes dimensiones de beneficios (cesación, salud individual y salud de terceros). Se concluyó que los fumadores mexicanos que desean dejar de fumar atribuyen un alto valor monetario por un método de cesación efectivo. Los fumadores del sexo masculino evidenciaron una actitud menos altruista que las mujeres fumadoras y se demostró una fuerte asociación entre la DAP y los niveles educativo y socioeconómico.

El objetivo del presente estudio fue identificar factores socioeconómicos, demográficos, de la historia de tabaquismo y contextuales asociados con el deseo de dejar de fumar, estimar la DAP por un TCT efectivo e identificar los asociados con esta valoración.

\section{Material y métodos}

La información analizada se obtuvo a partir de la Encuesta Global de Tabaquismo en Adultos (GATS) México 2009. Se trata de una encuesta de representatividad nacional ( $\mathrm{N}=13627)$, por sexo y tamaño de localidad, cuyo propósito fue recolectar, entre la población mayor de 15 años, información sobre consumo de tabaco, experiencia en el uso de TCT, exposición a humo de tabaco, características socioeconómicas y demográficas, conocimiento y actitudes acerca de la epidemia, y las percepciones sobre las estrategias de control en las advertencias sanitarias y en los medios de comunicación.

Adicionalmente, la GATS-México incluyó preguntas sobre la DAP de los fumadores por un TCT. Las preguntas de esta sección fueron agregando progresivamente información sobre los beneficios potenciales del TCT en tres dimensiones de bienestar: cesación, mejoras en la salud individual y mejoras en la salud familiar. La DAP por un TCT se identificó mostrando a los individuos un listado de valores prefijados y ordenados (a partir de precios nacionales de TCT), entre los cuales los participantes eligieron su respuesta (apéndice I). El protocolo de investigación que permitió el desarrollo de

\footnotetext{
* Heredia-Pi IB, Serván-Mori E, Reynales-Shigematsu LM, BautistaArredondo S. The Maximum Willingness to Pay for Smoking Cessation Method among Adult Smokers in Mexico. Value in Health 2012. En prensa.
}

la GATS en México fue aprobado por las Comisiones de Investigación y de Ética del Instituto Nacional de Salud Pública, y durante el desarrollo de la encuesta se obtuvo el consentimiento informado de los sujetos participantes en el estudio.

La muestra de análisis incluyó 1626 fumadores actuales (diario $\mathrm{u}$ ocasional), divididos entre aquellos que deseaban o no dejar de fumar (fumador diario $\mathrm{u}$ ocasional que responde afirmativamente/negativamente a la pregunta ¿Usted quiere dejar de fumar?). A quienes respondieron afirmativamente $(\mathrm{N}=1043)$ se les aplicaron las preguntas relacionadas con su disponibilidad a pagar.

En primer lugar caracterizamos a los fumadores actuales con base en variables socioeconómicas, demográficas, de historia de tabaquismo y de entorno. Entre estas variables se encuentran la edad del fumador (15-24, 25-44, 45-64 y 65 y más años de edad); nivel educativo (sin educación formal, primaria, secundaria, preparatoria, y licenciatura o superior); condición laboral (si trabajaba o no); tipo de localidad de residencia (urbana, semiurbana y rural) y variables relacionadas con la historia de tabaquismo de los individuos (tipo de fumador -diario, adicto o no y ocasional-; número de años de fumar; conocimientos sobre centros de apoyo al fumador y el antecedente de intentos previos de cesación). Adicionalmente, se identificaron las principales diferencias entre los fumadores de acuerdo con su disposición para dejar de fumar.

En segundo lugar, identificamos los factores asociados con el deseo de cesación, tanto para quienes han intentado dejar de fumar como para aquellos que no, con base en la estimación de modelos logit. Dichos factores incluyeron, además de las características señaladas anteriormente, un índice de nivel socioeconómico (NSE) de los individuos, el cual conjuga, a partir de un análisis de componentes principales con matrices de correlación policórica, ${ }^{24-29}$ la posesión de diferentes tipos de activos y características de la infraestructura de los hogares. El apéndice II detalla las variables utilizadas en la construcción del índice de NSE. Los resultados del modelo logit fueron expresados en razones de momios.

Finalmente, analizamos la DAP (en pesos de 2009) por un TCT a partir de dos aproximaciones. La primera, con base en un análisis gráfico, a través del cual mostramos diferencias en DAP por sexo, NSE, nivel educativo, años de fumar, conocimiento de centros de apoyo e intentos previos por dejar de fumar. La segunda identifica los factores asociados con la DAP en escala logarítmica. Ambos análisis se realizaron con base en modelos de regresión lineal múltiples que incluyeron errores estándar robustos. En particular, en el caso de la segunda estrategia se estimaron modelos de asociación 
estadística aditivos con el fin analizar la robustez de las estimaciones. El análisis se realizó utilizando el software STATA SE 11.0.

\section{Resultados}

El cuadro I muestra las características sociodemográficas, demográficas y de tabaquismo en la muestra de fumadores analizada, así como las diferencias entre aquellos que desean dejar de fumar y los que no. Entre el grupo que no deseaba abandonar el consumo de productos de tabaco, una mayor proporción fueron hombres. Otras diferencias se detectaron en edad, nivel educativo, NSE, localidad de residencia, tipo de fumador y la experiencia previa de intentos previos para dejar de fumar.

El cuadro II muestra los factores asociados con la probabilidad de querer dejar de fumar para dos grupos de fumadores (con y sin intentos previos de cesación). Los hombres sin intentos previos tienen menor probabilidad de querer dejar de fumar $(\mathrm{RM}=0.622)$ que las

Cuadro I

Características individuales Sociodemográficas y Sobre la eXPeriencia tabáQuica,

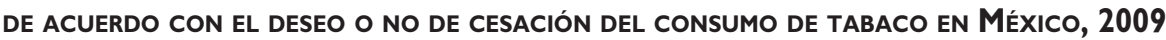

\begin{tabular}{|c|c|c|c|c|}
\hline & $\begin{array}{c}\text { Todos } \\
\text { media (DE) } \\
(N=1 \quad 626)\end{array}$ & $\begin{array}{c}\text { No desea cesar } \\
\text { media }(D E) \\
(N=583)\end{array}$ & $\begin{array}{l}\text { Desea cesar } \\
\text { media (DE) } \\
(N=1043)\end{array}$ & $\begin{array}{c}\text { Diferencia de medias } \\
\text { o proporciones } \\
\text { valor } p^{*}\end{array}$ \\
\hline Sexo (Hombre) & 0.775 & 0.822 & 0.749 & 0.000 \\
\hline \multicolumn{5}{|l|}{ Edad } \\
\hline $15-24$ & 0.328 & 0.346 & 0.318 & 0.001 \\
\hline $25-44$ & 0.442 & 0.425 & 0.452 & \\
\hline $45-64$ & 0.175 & 0.148 & 0.191 & \\
\hline 65 y más & 0.054 & 0.081 & 0.039 & \\
\hline \multicolumn{5}{|l|}{ Nivel educativo } \\
\hline Sin educación formal & 0.204 & 0.184 & 0.216 & 0.071 \\
\hline Primaria & 0.249 & 0.223 & 0.264 & \\
\hline Secundaria & 0.312 & 0.329 & 0.302 & \\
\hline Preparatoria & 0.162 & 0.180 & 0.151 & \\
\hline Licenciatura o superior & 0.073 & 0.084 & 0.067 & \\
\hline Trabaja & 0.708 & 0.720 & 0.702 & 0.427 \\
\hline \multicolumn{5}{|l|}{ Nivel socioeconómico } \\
\hline Quintil I & 0.127 & 0.144 & 0.118 & 0.031 \\
\hline Quintil 2 & 0.156 & 0.134 & 0.169 & \\
\hline Quintil 3 & 0.198 & 0.170 & 0.214 & \\
\hline Quintil 4 & 0.236 & 0.250 & 0.228 & \\
\hline Quintil 5 & 0.282 & 0.302 & 0.271 & \\
\hline \multicolumn{5}{|l|}{ Localidad de residencia } \\
\hline Urbana & 0.351 & 0.310 & 0.373 & 0.039 \\
\hline Semi - urbana & 0.259 & 0.278 & 0.248 & \\
\hline Rural & 0.391 & 0.412 & 0.379 & \\
\hline \multicolumn{5}{|l|}{ Antecedentes tabáquicos } \\
\hline Fumador ocasional & 0.472 & 0.417 & 0.502 & 0.001 \\
\hline Fumador diario & 0.528 & 0.583 & 0.498 & \\
\hline Adicto & 0.145 & 0.139 & 0.147 & 0.797 \\
\hline No adicto & 0.855 & 0.861 & 0.853 & \\
\hline Número de años fumando & $16.62(14.65)$ & $17.28(15.68)$ & $16.25(14.04)$ & 0.189 \\
\hline Conoce centros de apoyo para dejar de fumar & 0.101 & 0.106 & 0.098 & 0.587 \\
\hline Intentos previos para dejar de fumar & 0.442 & 0.214 & 0.57 & 0.000 \\
\hline
\end{tabular}

Nota:

* Se reportan las diferencias entre el grupo que desea cesar y el que no desea cesar. Para el caso de las variables categóricas se reporta el valor p del estadístico $\chi^{2}$ de Pearson. Para el caso de variables continuas se reporta el valor $p$ del estadístico $t$

Fuente: Elaboración propia de los autores a partir de los datos de la GATS 2009 
Cuadro II

Factores asociados con el deseo de cesar el consumo de tabaco, SEGÚN historia de INTENTOS PREVIOS de CESACIÓN. MéXico, 2009

\begin{tabular}{|c|c|c|}
\hline & \multicolumn{2}{|c|}{ Desea cesar= I, Razones de momios reportados } \\
\hline & Sin intentos previos & Con intentos previos \\
\hline \multirow{2}{*}{ Hombre } & $0.622 *$ & 0.740 \\
\hline & {$[0.422-0.916]$} & {$[0.437-1.255]$} \\
\hline \multicolumn{3}{|c|}{ Edad (Ref.: 15 - 24 años) } \\
\hline \multirow{2}{*}{$25-44$} & 1.220 & $1.925^{*}$ \\
\hline & {$[0.810-1.838]$} & [1.028 - 3.607] \\
\hline \multirow{2}{*}{$45-64$} & 1.510 & $5.022 *$ \\
\hline & {$[0.692-3.293]$} & {$[1.358-18.574]$} \\
\hline \multirow[b]{2}{*}{65 y más } & 0.807 & 1.088 \\
\hline & {$[0.225-2.894]$} & {$[0.172-6.859]$} \\
\hline
\end{tabular}

Nivel educativo (Ref.: Ninguno)

\begin{tabular}{lcr} 
Primaria & 1.223 & $0.418^{*}$ \\
\hline \multirow{2}{*}{ Secundaria } & {$[0.801-1.868]$} & {$[0.194-0.900]$} \\
\hline \multirow{2}{*}{ Preparatoria } & 0.740 & $0.343^{\ddagger}$ \\
\hline \multirow{2}{*}{ Más que preparatoria } & {$[0.476-1.148]$} & {$[0.157-0.749]$} \\
\hline \multirow{2}{*}{ Trabaja } & 0.711 & $\left.0.43\right|^{\S}$ \\
\cline { 2 - 3 } & {$[0.424-1.194]$} & $0.177-1.051]$ \\
\cline { 2 - 3 }
\end{tabular}

Nivel socioeconómico (Ref.: Quintil I)

Quintil 2

I.19|

$2.201^{\S}$

Q Q

Quintil 3

Quintil 4

Quintil 5

\begin{tabular}{cc}
1.191 & $\left.2.20\right|^{\S}$ \\
\hline$[0.712-1.993]$ & {$[0.968-5.005]$} \\
\hline 1.085 & $3.392^{\ddagger}$ \\
\hline$[0.651-1.809]$ & {$[1.462-7.871]$} \\
\hline 1.106 & 1.399 \\
\hline$[0.663-1.847]$ & {$[0.653-2.996]$} \\
\hline 1.159 & 1.536 \\
\hline$[0.685-1.961]$ & {$[0.686-3.440]$}
\end{tabular}

Localidad de residencia (Ref.: Urbana)

\begin{tabular}{lcc} 
Semi - urbana & 0.789 & 0.627 \\
\cline { 2 - 3 } Rural & {$[0.542-1.148]$} & {$[0.355-1.106]$} \\
\cline { 2 - 3 }
\end{tabular}

Antecedentes tabáquicos

Tipo de fumador (Ref.: ocasional)

\begin{tabular}{|c|c|c|}
\hline & & \\
\hline Adicto & $2.039 *$ & 0.760 \\
\hline HUICLO & {$[\mathrm{I} .15 \mathrm{I}-3.6 \mathrm{II}]$} & {$[0.339-1.706]$} \\
\hline & $1.777^{\ddagger}$ & 1.202 \\
\hline Diario no adicto & {$[1.330-2.375]$} & {$[0.768-1.88 I]$} \\
\hline & 0.981 & $0.98 I$ \\
\hline Número de años fumando & {$[0.959-1.004]$} & {$[0.947-1.016]$} \\
\hline & 1.137 & 0.644 \\
\hline Conoce centros de apoyo para dejar de fumar & {$[0.7 \mid I-I .8 I 7]$} & {$[0.361-1.148]$} \\
\hline Observaciones & 907 & 719 \\
\hline Log de verosimilitud & -604.9 & -305.1 \\
\hline Pseudo $\mathrm{R}^{2}$ & 0.038 & 0.081 \\
\hline $\mathrm{AIC}$ & 1,250 & 650.2 \\
\hline
\end{tabular}

Nota: Intervalos de confianza al $95 \%$ en corchetes

$* p<0.05$

$\ddagger p<0.01$

$\S p<0.1$

Fuente: Elaboración propia de los autores a partir de los datos de la GATS 2009 
fumadoras del sexo femenino. Entre los que reportaron haber intentado dejar de fumar previamente, al compararse frente al grupo de edad de 15-24 años, la RM estimada para el grupo de 25-44 años fue de 1.925 y para el grupo de 45-64 años, dicha RM fue 5.022. En este mismo grupo y, frente a quintil 1 de NSE, la RM del quintil 3 fue 3.392. Finalmente, las características de la historia del fumador resultaron estadísticamente significativas entre los fumadores sin intentos previos de cesación. En este grupo, la RM para los fumadores adictos fue 2.039.

El cuadro III muestra las principales diferencias en las características señaladas anteriormente entre los fumadores, según su DAP. Quienes manifestaron una DAP $>0$ fueron los más jóvenes, con mejor nivel educativo, aquellos residentes en localidades rurales y quienes reportaron intentos previos de cesación. En

\section{Cuadro III}

Perfil de los fumadores en México, 2009, según su disponibilidad a pagar por un método de cesación tabáquica

\begin{tabular}{|c|c|c|c|}
\hline & $\begin{array}{c}\text { Disponibilidad a pagar }=0 \\
\text { media }(\mathrm{DE}) \\
(\mathrm{N}=199)\end{array}$ & $\begin{array}{c}\text { Disponibilidad a pagar }>0 \\
\text { media (DE) } \\
(N=844)\end{array}$ & $\begin{array}{c}\text { Diferencia de medias } \\
\text { o proporciones } \\
\text { valor } \mathrm{p}^{*}\end{array}$ \\
\hline Hombre & 0.749 & 0.749 & 0.998 \\
\hline \multicolumn{4}{|l|}{ Edad } \\
\hline $15-24$ & 0.276 & 0.328 & 0.093 \\
\hline $25-44$ & 0.432 & 0.456 & \\
\hline $45-64$ & 0.231 & 0.181 & \\
\hline 65 y más & 0.060 & 0.034 & \\
\hline \multicolumn{4}{|l|}{ Nivel educativo } \\
\hline Ninguno & 0.276 & 0.201 & 0.017 \\
\hline Primaria & 0.312 & 0.252 & \\
\hline Secundaria & 0.246 & 0.315 & \\
\hline Preparatoria & 0.111 & 0.161 & \\
\hline Más que preparartoria & 0.055 & 0.070 & \\
\hline Trabaja & 0.688 & 0.705 & 0.650 \\
\hline \multicolumn{4}{|l|}{ Nivel socioeconómico } \\
\hline Quintil I & 0.116 & 0.118 & 0.996 \\
\hline Quintil 2 & 0.176 & 0.167 & \\
\hline Quintil 3 & 0.216 & 0.213 & \\
\hline Quintil 4 & 0.231 & 0.227 & \\
\hline Quintil 5 & 0.261 & 0.274 & \\
\hline \multicolumn{4}{|l|}{ Localidad de residencia } \\
\hline Urbana & 0.372 & 0.373 & 0.065 \\
\hline Semi - urbana & 0.307 & 0.235 & \\
\hline Rural & 0.322 & 0.392 & \\
\hline \multicolumn{4}{|l|}{ Antecedentes tabáquicos } \\
\hline Fumador ocasional & 0.462 & 0.512 & 0.209 \\
\hline Fumador diario & 0.538 & 0.488 & \\
\hline Adicto & 0.141 & 0.148 & 0.866 \\
\hline No adicto & 0.859 & 0.852 & \\
\hline Número de años fumando & $18.23(14.53)$ & $15.78(13.89)$ & 0.031 \\
\hline Conoce centros de apoyo para dejar de fumar & 0.101 & 0.097 & 0.888 \\
\hline Intentos previos para dejar de fumar & 0.497 & 0.586 & 0.024 \\
\hline Disponibilidad a pagar (en miles de pesos) & - & $2.708(4.439)$ & - \\
\hline
\end{tabular}

Fuente: Elaboración propia de los autores a partir de los datos de la GATS 2009 
promedio, la DAP fue 2708 pesos (USD \$ 200.6). Por su parte, quienes manifestaron una $\mathrm{DAP}=0$ fueron quienes tenían más años de fumar.

El análisis gráfico de la DAP muestra que existen diferencias significativas por sexo, NSE y nivel educativo. No existieron diferencias por experiencia tabáquica ni por conocimiento de centros de apoyo (figura 1). Finalmente, los modelos multivariados presentados en el cuadro IV (modelo 3) confirman los resultados del análisis gráfico; el nivel de educación y el NSE se asociaron a una mayor DAP.

\section{Discusión}

A partir de la caracterización socioeconómica, demográfica, de tabaquismo y del entorno de los fumadores mexicanos de 15 y más años de edad, nuestro estudio presenta resultados relevantes para el diseño e implementación de intervenciones de apoyo a los fumadores durante su intento por abandonar el consumo de tabaco. La utilidad de la información generada por este estudio es relevante porque identifica tres niveles de oportunidad para focalizar intervenciones de cesación tabáquica.

En primer lugar, se caracterizó a dos grupos de fumadores (los que desean y los que no desean dejar de fumar). Estos grupos se diferencian por variables como sexo, nivel educativo, NSE, localidad de residencia, tipo de fumador (diario u ocasional), años de fumar e intentos previos de cesación. Ello evidencia que existe una población de fumadores sobre quienes hay necesidad de dirigir estrategias de cesación más enérgicas e integrales
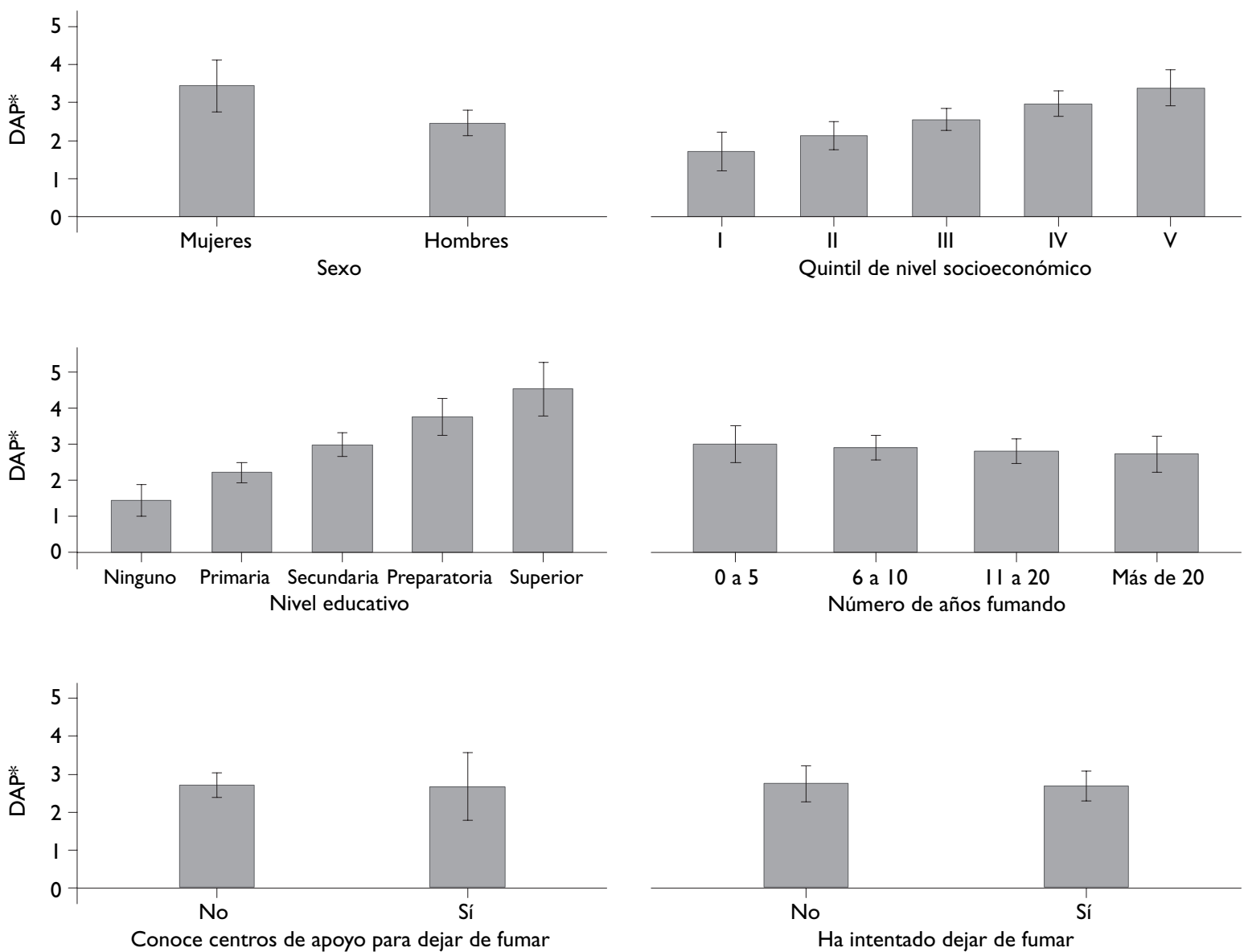

\footnotetext{
* Significa disponibilidad a pagar (en miles de pesos mexicanos). Intervalos de confianza al 95\%. Estimaciones ajustadas por sexo, nivel educativo, nivel socioeconómico, número de años fumando, conocimiento sobre centros de apoyo para dejar de fumar e intentos previos para abandonar la adicción
}

Fuente: Elaboración propia de los autores a partir de los datos de la GATS 2009

Figura I. Diferencias en la disponibilidad a pagar por un tratamiento de CeSACión tabáQUica según características SOCIODEMOGRÁFICAS Y EXPERIENCIA TABÁQUICA ENTRE FUMADORES QUE DESEAN DEJAR DE CONSUMIR TABACO EN MÉXICO, 2009 


\section{Cuadro IV}

FACTORES ASOCIADOS CON EL MONTO dE LA DISPONIBILIDAd A PAGAR POR UN TRATAMIENTO DE CESACIÓN TABÁQUICA,

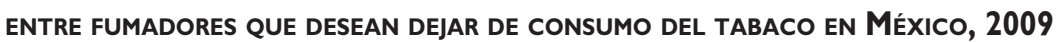

\begin{tabular}{|c|c|c|c|}
\hline & & dad a pagar (er & \\
\hline & Modelo I & Modelo 2 & Modelo 3 \\
\hline & $-0.389 *$ & $-\left.0.31\right|^{\S}$ & -0.268 \\
\hline Hombre & {$[0.159]$} & {$[0.174]$} & {$[0.178]$} \\
\hline Edad & & & \\
\hline $25-44$ & -0.029 & -0.020 & 0.160 \\
\hline $2 b-44$ & {$[0.152]$} & {$[0.150]$} & {$[0.194]$} \\
\hline & -0.272 & -0.105 & 0.324 \\
\hline $4 b-64$ & {$[0.200]$} & {$[0.199]$} & {$[0.381]$} \\
\hline 65 y más & $\begin{array}{l}-\left.1.34\right|^{\ddagger} \\
{[0.309]}\end{array}$ & $\begin{array}{c}-0.704 * \\
{[0.307]}\end{array}$ & $\begin{array}{c}0.010 \\
{[0.613]}\end{array}$ \\
\hline Nivel educativo & & & \\
\hline Primaria & & $0.485^{*}$ & $0.459 *$ \\
\hline & & {$[0.192]$} & {$[0.193]$} \\
\hline & & $0.746^{\ddagger}$ & $0.693^{\ddagger}$ \\
\hline Secundaria & & {$[0.200]$} & {$[0.200]$} \\
\hline Prenaratoria & & $0.835^{\ddagger}$ & $0.808^{\ddagger}$ \\
\hline Preparatorla & & {$[0.247]$} & {$[0.249]$} \\
\hline 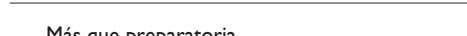 & & $1.433^{\ddagger}$ & $1.403^{\ddagger}$ \\
\hline Mas que preparatorla & & {$[0.3 \mid 3]$} & {$[0.315]$} \\
\hline Trabaja & & 0.136 & 0.131 \\
\hline & & {$[0.160]$} & {$[0.159]$} \\
\hline Nivel socioeconómico & & & \\
\hline Ouintil 2 & & 0.314 & 0.319 \\
\hline Quintil 2 & & {$[0.242]$} & {$[0.244]$} \\
\hline Ouintil 3 & & $0.447+$ & $0.46 I^{*}$ \\
\hline Quintil 3 & & {$[0.231]$} & {$[0.232]$} \\
\hline & & $0.779 \ddagger$ & $0.782^{\ddagger}$ \\
\hline Quintil 4 & & {$[0.240]$} & {$[0.242]$} \\
\hline Quintil 5 & & $\begin{array}{r}0.698^{\ddagger} \\
{[0.244]}\end{array}$ & $\begin{array}{r}0.71 \mathrm{I}^{\ddagger} \\
{[0.248]}\end{array}$ \\
\hline Localidad de residencia & & & \\
\hline & & 0.037 & 0.029 \\
\hline semiurbana & & {$[0.182]$} & {$[0.183]$} \\
\hline Rural & & -0.179 & -0.197 \\
\hline Nuld & & {$[0.170]$} & {$[0.171]$} \\
\hline Antecedentes tabáquicos & & & \\
\hline Tipo de fumador (Ref.: ocasional) & & & \\
\hline I & & & -0.006 \\
\hline Adicto & & & {$[0.257]$} \\
\hline Diarin no adicto & & & 0.208 \\
\hline Diarıo no adicto & & & {$[0.143]$} \\
\hline Número de años fumando & & & -0.017 \\
\hline Número de años fumando & & & {$[0.011]$} \\
\hline & & & 0.046 \\
\hline Conoce centros de apoyo para dejar de fumar & & & {$[0.229]$} \\
\hline Intentos previos para dejar de fumar & & & $\begin{array}{l}-0.056 \\
{[0.136]}\end{array}$ \\
\hline Intercepto & $\begin{array}{c}-0.233 \\
{[0.166]}\end{array}$ & $\frac{-\left.1.49\right|^{\ddagger}}{[0.256]}$ & $\begin{array}{c}-1.474^{\ddagger} \\
{[0.276]}\end{array}$ \\
\hline Observaciones & 844 & 844 & 844 \\
\hline $\mathrm{R}^{2}$ & 0.023 & 0.084 & 0.090 \\
\hline AIC & 3,524 & 3,492 & 3,496 \\
\hline Estadísticos del error estimado & & & \\
\hline Promedio & 0.000 & 0.000 & 0.000 \\
\hline Kurtosis & 0.274 & 0.223 & 0.234 \\
\hline Skewness & 1.851 & 2.000 & 2.003 \\
\hline $\begin{array}{l}\text { Nota: Modelos estimados por Mínimos Cuad } \\
* p<0.05 \\
\ddagger p<0.01 \\
\S p<0.1\end{array}$ & robustos e & & \\
\hline Fuente: Elaboración propia de los autores a p & & & \\
\hline
\end{tabular}


que los motiven a querer dejar de fumar y que podrían dirigirse de manera específica (aunque con componentes diferenciados de acuerdo con la edad) hacia fumadores hombres, de bajo NSE, residentes de localidades rurales y semiurbanas que fumen diariamente, con mayor tiempo de ser fumadores y sin intentos previos de cesación. Según la expansión poblacional de nuestros resultados, este grupo poblacional representa 37.75\% (4095 993) de los fumadores mexicanos actuales.

En segundo lugar, de acuerdo con nuestros resultados, entre los fumadores que ya desean dejar de fumar se pueden identificar dos grupos: aquellos con disponibilidad a pagar (DAP) por un tratamiento de cesación tabáquica (TCT) igual a 0 y aquellos con DAP $>0$. Estos últimos representan $84.04 \%$ (5676 755) de los fumadores interesados en abandonar el consumo de productos de tabaco. Para estos dos grupos se identificó igualmente un perfil diferenciado en función de la edad, nivel educativo, número de años de fumar y la existencia de intentos previos de cesación. Esta información puede ser útil al pensar en intervenciones de cesación diferenciadas en función de su financiamiento: intervenciones de cesación gratuitas para todos los fumadores (cobertura universal para todos los fumadores recomendada por la $\left.\mathrm{OMS}^{30}\right)$, versus intervenciones con mecanismos de pago que permitan aprovechar la disponibilidad a pagar y la capacidad de pago de algunos fumadores.

Finalmente, nuestros resultados muestran que entre los fumadores que tienen una DAP positiva, la cantidad que estarían dispuestos a pagar se asocia con la edad, el nivel educativo y el NSE de los individuos. No sólo corroboran dicha relación ya reportada en un estudio previo, ${ }^{19}$ sino que se evidencia la existencia de una asociación creciente entre nivel educativo y la DAP de los fumadores mexicanos. ${ }^{19,20}$ Nuestros resultados sugieren además que en México el nivel educativo se asocia de manera más fuerte que el NSE con una mayor DAP. Se encontraron resultados similares en un estudio previo realizado a partir de esta misma encuesta, aunque con una muestra analítica distinta. Por otra parte, la asociación entre la DAP y las variables relacionadas con la historia de fumador en el presente análisis no mostró ser estadísticamente significativa, a diferencia de lo informado en estudios previos, donde la intensidad del consumo de cigarrillos y los intentos previos de cesación se asocian significativamente con la DAP reportada por los fumadores. ${ }^{20}$

En promedio, la DAP de los fumadores mexicanos fue 2708 pesos mexicanos por TCT. Si comparamos este valor y los precios actuales de este tipo de tecnología disponible en México, como por ejemplo, los parches de nicotina (medicamentos incorporados al cuadro básico del sector salud mexicano), ${ }^{*}$ la DAP estimada fue aproximadamente $60 \%$ superior al costo real del tratamiento completo (considerando los precios de compra del Instituto Mexicano del Seguro Social (IMSS) para el año 2009). ${ }^{31}$ Esto podría estar sugiriendo la existencia de una demanda potencial aún no cubierta por la oferta actual de tratamientos para dejar de fumar en los servicios de salud en México.

Esta información sugiere que existe una ventana de oportunidad para establecer mecanismos de financiamiento mixtos o de copagos, donde tanto los fumadores como el gobierno aporten recursos para financiar intervenciones de cesación. Una posibilidad, por ejemplo, puede ser establecer mecanismos de cofinanciamiento para aquellas tecnologías más novedosas y de elevado costo. Una extensa literatura sobre el efecto crowding-out en el sistema de salud estadounidense deja importantes lecciones que se pueden aprovechar para financiar de manera privada algunos bienes de salud, en vez de desplazar el financiamiento público de otros bienes que sí deben ser financiados de manera pública. ${ }^{32,33}$

Tomar en cuenta los resultados de nuestro estudio puede hacer factible el propósito a corto y mediano plazo de encaminar al sistema de salud mexicano a lograr que las intervenciones de cesación tabáquica se oferten en los tres niveles de atención y en todas las instituciones de salud del sistema de salud mexicano, tanto públicas como privadas. Adicionalmente, se pueden implementar otras acciones que generen sinergias importantes en el proceso de implementación de las políticas de cesación en el contexto nacional, estableciendo algoritmos de prevención y de tratamiento diferenciados de acuerdo a los perfiles identificados en el presente estudio.

Éste no está exento de limitaciones. En el análisis aquí presentado puede existir un potencial sesgo de selección debido a que sólo se tiene información sobre la DAP para aquellos fumadores que deseaban dejar de fumar. Este es un problema de origen de la encuesta, ya que no se preguntó sobre la DAP a quienes no estaban dispuestos a dejar de fumar. El efecto de este inconveniente sobre nuestras estimaciones no es claro, $y$ futuras encuestas deberán considerar este aspecto. Otra limitante es que las preguntas de la encuesta hacen referencia a un TCT 100\% efectivo; sin embargo, sabemos que en la práctica no existe ningún TCT perfectamente efectivo, lo cual podría conllevar una sobreestimación de nuestros resultados. Finalmente, sólo comparamos

\footnotetext{
* Listado de medicamentos que se requieren en las instituciones del sector salud para atender los principales problemas de salud de la población mexicana.
} 
la DAP de los fumadores con los precios publicados por el IMSS y reconocemos que los precios de compra de las otras instituciones del sector salud mexicano no necesariamente corresponden con los precios de compra de esta institución.

\section{Agradecimientos}

La Encuesta Global de Tabaquismo en Adultos (GATS) recibió financiamiento de la Iniciativa Bloomberg para reducir el consumo de tabaco, un programa de Filantropías Bloomberg. Los patrocinadores no tuvieron ningún papel en el diseño, recolección y análisis de datos, ni en la decisión de la publicación o preparación de este manuscrito.

Declaración de conflicto de intereses. Los autores declararon no tener conflicto de intereses.

\section{Referencias}

I. World Health Organization. WHO Framework Convention on Tobacco Control. Geneva: World Health Organization, 2003.

2. WHO Report on the Global Tobacco Epidemic, 2008 - The MPOWER package. [Consultado 12 de agosto de 2009] Disponible en: http://www. who.int/tobacco/mpower/en/.

3. Regalado-Pineda J, Lara-Rivas G, Osio-Echánove J, Ramírez-Venegas A. Tratamiento actual del tabaquismo. Salud Publica Mex 2007;49(supl 2):S270-S279.

4. Sansores RH, Ramírez-Venegas A, Espinosa- Martínez M, Sandoval RA. Tratamientos para dejar de fumar disponibles en México. Salud Publica Mex 2002;44( SI):II6-I24.

5. Jorenby DE, Leischow SJ, Nides MA, Rennard SI, Johnston JA, Hughes $A R$ et al. A controlled trial of sustained-release bupropion, a nicotine patch, or both for smoking cessation. N Engl J Med 1999;340(9):685-69I. 6. Oncken C, Gonzales D, Nides M, Rennard S, Watsky E, Billing CB, et al. Efficacy and Safety of the Novel Selective Nicotinic Acetylcholine Receptor Partial Agonist, Varenicline, for Smoking Cessation. Arch Intern Med 2006; 166:157|-1577.

7. West R. $A B C$ of smoking cessation. Assessment of dependence and motivation to stop smoking. BMJ 2004;328:338-339.

8. Encuesta Nacional de Adicciones 2008. Instituto Nacional de Salud Pública, Cuernavaca, México. [Consultado: 2 de noviembre de 2009]. Disponible en http://www.insp.mx/Portal//nf/ENA08_nacional.pdf. 9. Organización Panamericana de la Salud, Instituto Nacional de Salud Pública (MX). Encuesta Global de Tabaquismo en Adultos. México 2009. Cuernavaca (México): Instituto Nacional de Salud Pública, 2010. Coeditado con la Organización Panamericana de la Salud, 2010. [Consultado: 8 de octubre de 2010]. Disponible en: http://www.insp.mx/ images/stories/INSP/Docs/GATS.pdf.

10. US Department of Health and Human Services. The Health Benefits of Smoking Cessation. A report of the Surgeon General, 1990. Department of Health and Human Services, Centers for Disease Control and Prevention, National Center for Chronic Disease Prevention and Health Promotion, Office on Smoking and Health. [Consultado: 20 de mayo de 2008]. Disponible en: http://profiles.nlm.nih.gov/NN/B/B/C/T/_I nnbbct.pdf.

II. Centers for Disease Control and Prevention. Smoking cessation during previous year among adults-United States, 1990 and 1991. Morb Mortal Wkly Rep 1993;42:504-507.
12. González-Enríquez J, Salvador-Llivina T, López-Nicolás A, Antón de las Heras E, Musin A, Fernández E, et al. Morbilidad, mortalidad y costes sanitarios evitables mediante una estrategia de tratamiento del tabaquismo en España. Gac Sanit 2002;16(4):308-317.

13. Antoñanzas $F$, Portillo $F$. Evaluación económica del empleo de terapias farmacológicas para la cesación en el hábito tabáquico. Gac Sanit 2003; I7(5):393-403

14. Arrow K, Solow R, Portney PR, Leamer EE, Radner R, Schuman H. Report of the NOAA panel of contingent valuation. Federal Register 1993;58:460I-46I4.

15. Smith RD Sach TH. Contingent Valuation: (Still) on the Road to nowhere? Health Econ 2009; 18:863-866.

16. Frew E, Wolstenholme JL, Whynes DK. Willingness-to-pay for colorectal cancer screening. Eur J Cancer 200 I;37: 1746-175I.

17. Frew EJ, Whynes DK, Wolstenholme JL .Eliciting Willingness to Pay: Comparing Closed-Ended with Open-Ended and Payment Scale Formats. Med Decis Making 2003;23:150-159.

18. Whynes DK, Frew EJ, Wolstenholme JL. Willingness-to-Pay and Demand Curves: A Comparison of Results Obtained Using Different Elicitation Formats. Int J Health Care Finance Econ 2005;(5):369-386. 19. Busch SH, Falba TA, Duchovny N, Jafre-Bonet M, O'Malley SS, Sindelar JL. Value to smokers of improved cessation products: evidence from a willingness-to-pay survey. Nicotine Tob Res 2004:6(4):63 I-639. 20. Weimer DL, Vining AR, Thomas RK. Cost-Benefit Analysis involving addictive goods: contingent valuation to estimate willingness to pay for smoking cessation. Health Econ 2009; 18:181-202.

21. Avila-Burgos L, Gutiérrez-Zúñiga C, Hernández-Peña P, Santos-Burgoa C, Silvia-Aycaguer L. El costo social de la bronquitis crónica en la Ciudad de México: una experiencia piloto. Salud Publica Mex 1996; I28-138. 22. Vargas-Bustamante A, Ojeda G, Castañeda X. Willingness to pay for cross border health insurance between the United States and Mexicoel futuro Estados Unidos y México los programas de inmigración. Health Affairs 2008;27(I)I(2008):169-178;10.1377/hlthaff.27.I.169]:169-I78. 23. Vásquez WF, Mozumder P, Hernández-Arce J, Berrens RP. Willingness to pay for safe drinking water: Evidence from Parral, Mexico. J Environ Manage 2009;90(I I):339I-3400.

24. Kolenikov S, Angeles G. The use of discrete data in PCA: theory, simulations, and applications to socioeconomic indices. Chapel Hill: Carolina Population Center, University of North Carolina, 2004. 25. McKenzie, D. Measuring inequality with assets indicators. J Pop Econ 2005; 18:229-260.

26. Filmer D, Pritchett LH. Estimating wealth effect without expenditure data - or tears: an application to educational enrollments in states of India. Demography 200 I;38: I I5-132.

27. Hotelling $\mathrm{H}$. Analysis of a complex of statistical variables into principal components. J Educ Psychol 1933;24:417-44I,498-520.

28. Anderson TW. An Introduction to Multivariate Statistical Analysis, 3rd edn. New York: John Wiley and Sons, 2003.

29. Mardia KV, Kent JT, Bibby J. M. Multivariate Analysis. London: Academic Press, 1980.

30. World Health Organization. Tools for Advancing Tobacco Control in the XXlst century: Policy Recommendations for Smoking Cessation and Treatment of Tobacco Dependence. Tools for public health. WHO, 2003. [Consultado: 28 de septiembre de 20I I]. Disponible en: http:// www.who.int/tobacco/resources/publications/tobacco_dependence/en/ 31. Instituto Mexicano del Seguro Social. Cuadro Básico Institucional de Medicamentos. [Consultado: 9 de marzo de 2009]. Disponible en: http:// www.imss.gob.mx/transparencia/CuadrosBasicos/medicamentos.htm. 32. Cutler DM, Gruber J. Does Public Insurance Crowd Out Private Insurance? Q J Econ 1996; I I:391-430.

33. Dubay L, Kenney G. The Effects of Medicaid Expansions on Insurance Coverage of Children. Future Child 1996;6(I):152-161. 


\section{Apéndice I

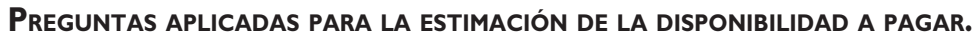 Encuesta Global de Tabaquismo en Adultos. México 2009}

Disponibilidad a pagar

Disponibilidad a pagar I (DAP I, en pesos mexicanos corrientes de 2009)

Disponibilidad a pagar 2 (DAP 2, en pesos mexicanos corrientes de 2009)

Disponibilidad a pagar 3 (DAP 3, en pesos mexicanos corrientes de 2009)
Descripción

Suponga que hay un método probado que le garantiza que si usted lo utiliza, va a poder dejar de fumar. El método no tiene consecuencias negativas para su salud, debe seguirlo por 3 meses, y le garantiza que usted no va a volver a fumar en los próximos 10 años. Por favor considere sus ingresos y los gastos que tiene que realizar de forma regular. Ahora mire las cantidades que aparecen en la tarjeta y dígame ¿cuánto sería lo máximo que estaría dispuesto a pagar por este método para dejar de fumar?

Respuesta: desde 0 a 15000 pesos mexicanos

Ahora piense en los beneficios que puede tener para su salud el dejar de fumar: se va a sentir más saludable, va a tener mejor condición física y va a reducir el riesgo de desarrollar algunas enfermedades como cáncer. Pensando en esto, e igual forma considerando sus ingresos y gastos, de las cantidades que aparecen en la tarjeta ¿cuánto sería lo máximo que estaría dispuesto a pagar por el método para dejar de fumar que le describí antes? Respuesta: desde 0 a 15000 pesos mexicanos
Ahora piense que si usted deja de fumar, la gente con la que vive y con la que convive de forma cotidiana puede resultar beneficiada porque ya no se expondrían al humo del cigarro y esto puede mejorar su salud. Pensando en esto, e igual forma considerando sus ingresos y sus gastos, de las cantidades que aparecen en la tarjeta ¿cuánto sería lo máximo que estaría dispuesto a pagar por el método para dejar de fumar que le describí antes?

Respuesta: desde 0 a 15000 pesos mexicanos 


\section{Apéndice II \\ VARIABLES INCLUIDAS EN LA CONSTRUCCIÓN DEL ÍNDICE SOCIOECONÓMICO}

Descripción

Tipo de variable

¿Algún integrante de esta vivienda cuenta con alguno de los siguientes elementos?

\begin{tabular}{|c|c|}
\hline Electricidad & Dicotómica $\left(S i ́=I, N_{0}=0\right)$ \\
\hline Inodoro de taza (baño, retrete) & Dicotómica $\left(\mathrm{Si}=\mathrm{I}, \mathrm{No}_{\mathrm{O}}=0\right)$ \\
\hline Teléfono fijo & Dicotómica $(\mathrm{Si}=\mathrm{I}, \mathrm{No}=0)$ \\
\hline Teléfono celular & Dicotómica $\left(S i=I, N_{0}=0\right)$ \\
\hline Televisión & Dicotómica $\left(\mathrm{Si}=\mathrm{I}, \mathrm{No}_{\mathrm{O}}=0\right)$ \\
\hline Radio & Dicotómica $(\mathrm{Si}=\mathrm{I}, \mathrm{No}=0)$ \\
\hline Refrigerador & Dicotómica $\left(S i=I, N_{0}=0\right)$ \\
\hline Automóvil & Dicotómica $\left(S i ́=I, N_{0}=0\right)$ \\
\hline Bici, moto, motoneta o motocicleta & Dicotómica $\left(S i ́=I, N_{0}=0\right)$ \\
\hline Lavadora automática & Dicotómica $(\mathrm{Si}=\mathrm{I}, \mathrm{No}=0)$ \\
\hline Tractor & Dicotómica $\left(S i ́=I, N_{0}=0\right)$ \\
\hline Camión & Dicotómica $\left(S i ́=I, N_{0}=0\right)$ \\
\hline $\begin{array}{l}\text { Otro tipo de vehículo como lancha, trajinera, } \\
\text { canoa }\end{array}$ & Dicotómica $(\mathrm{Si}=\mathrm{I}, \mathrm{No}=0)$ \\
\hline Calentador de gas para agua & Dicotómica $\left(S i ́=I, N_{0}=0\right)$ \\
\hline Computadora & Dicotómica $\left(S i ́=I, N_{0}=0\right)$ \\
\hline Horno de microondas & Dicotómica $(\mathrm{Si}=\mathrm{I}, \mathrm{No}=0)$ \\
\hline Licuadora & Dicotómica $\left(S i ́=I, N_{0}=0\right)$ \\
\hline DVD - videocasetera & Dicotómica $\left(S i ́=I, N_{0}=0\right)$ \\
\hline Ventilador & Dicotómica $\left(S i ́=I, N_{0}=0\right)$ \\
\hline ¿Qué tipo de sanitarios tiene su hogar? & $\begin{array}{l}\text { Variable dicotómica }(S i=\mid, N o=0) \text {. Se definió hol } 0=\mid \text { si el sanitario es un } \\
\text { desagüe al sistema de alcantarillado, desagüe o fosa séptica o letrina con } \\
\text { desagüe y hol } 0=0 \text { si el sanitario es una letrina seca cubierta o descubierta, } \\
\text { letrina de cubo o no hay sanitarios. }\end{array}$ \\
\hline $\begin{array}{l}\text { ¿De qué material es la mayor parte del piso de } \\
\text { esta vivienda? }\end{array}$ & $\begin{array}{l}\text { Variable dicotómica }(S i ́=I, N o=0) \text {. ho } 2=\mid \text { si el piso es de cemento o firme } \\
\text { o de mosaico, madera, u otros recubrimientos y ho } 2=0 \text { si el piso es de } \\
\text { tierra. }\end{array}$ \\
\hline $\begin{array}{l}\text { ¿De qué material es la mayor parte del techo de } \\
\text { esta vivienda? }\end{array}$ & $\begin{array}{l}\text { Variable dicotómica }(\mathrm{S} i=\mathrm{I}, \mathrm{No}=0) \text {. Se definió ho3=I si el techo es de teja, } \\
\text { losa de concreto o similar, tabique, ladrillo, tabicón o block y ho3=0 si el } \\
\text { techo es de cartón, hule, tela, llantas, lámina de cartón, palma, tejamanil, } \\
\text { madera, lámina metálica, fibra de vidrio, plástico, mica, lámina de asbesto, } \\
\text { carrizo, bambú o terrado. }\end{array}$ \\
\hline $\begin{array}{l}\text { ¿De qué material es la mayor parte de las paredes } \\
\text { o muros de esta vivienda? }\end{array}$ & $\begin{array}{l}\text { Variable dicotómica }(S i ́=I, N o=0) \text {. Se definió ho4=I si las paredes son de } \\
\text { cemento, ladrillo, piedra, madera o ladrillo de barro y ho } 4=0 \text { si las paredes } \\
\text { son de paja y similares, lámina de plástico u hoja de metal. }\end{array}$ \\
\hline
\end{tabular}

